Thorax (1966), 21, 48.

\title{
Cineradiography in the post-operative control of a Starr aortic valve prosthesis
}

\author{
LARS BJöRK AND VIKING OLOV BJÖRK \\ From the Departments of Diagnostic Radiology and Thoracic Surgery, University Hospital, \\ Uppsala, Sweden
}

In our series of 105 patients in whom a diseased aortic valve has been replaced by a Starr ball valve type prosthesis, there have been 12 cases of post-operative infection with a positive culture of bacteria from the blood. In eight patients the infection was the direct cause of death. Five of those who died within 55 to 144 days after the operation had a suture insufficiency around the prosthesis with regurgitation on the side of the ball valve prosthesis and a false aneurysm of the sinus of Valsalva (Fig. 1). Reoperation in two patients with exchange of the prosthesis and

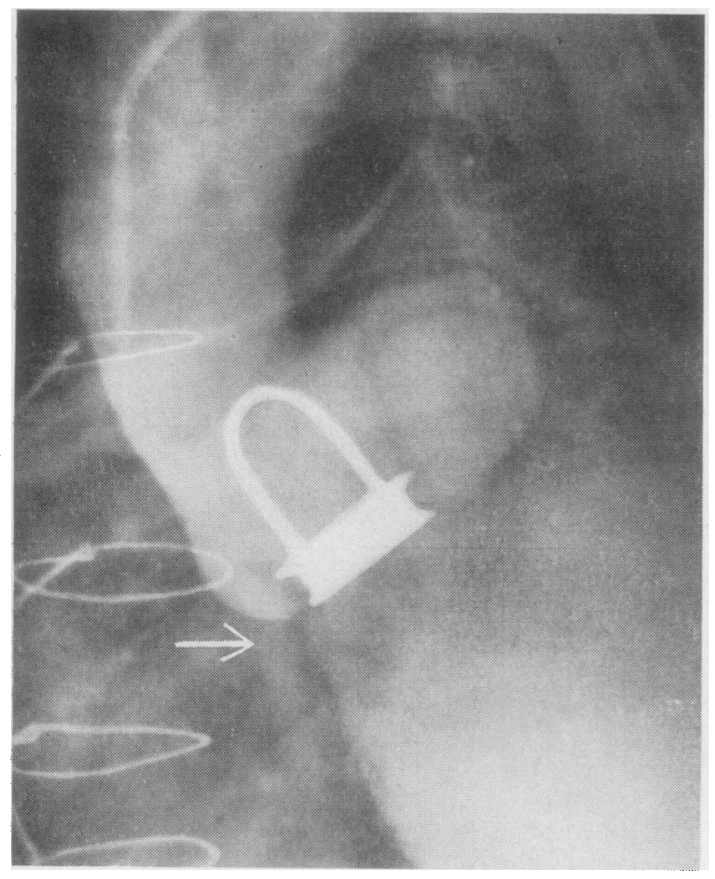

FIG. 1. Thoracic aortography in a patient with suture insufficiency around the base of the Starr ball valve prosthesis. Leakage of contrast medium into the left ventricle and a false aneurysm of the sinus of Valsalva (arrow). obliteration of the aneurysm of the sinus of Valsalva was unsuccessful. However, a suture insufficiency without infection was reoperated successfully in three patients.

This condition may, of course, be suspected or diagnosed clinically. For an early and definite diagnosis, however, a thoracic aortogram is usually necessary. The aortogram may have to be repeated two or three times before the diagnosis can be definitely established. This procedure causes the patient discomfort and carries a possible risk of thrombosis of the vessel used for introduction of the catheter.

In view of these facts we have looked for a simpler method objectively to study the function of the Starr prosthesis post-operatively. Since the metal cage of the prosthesis is visible on radiographs, cineradiography has been used to study the movements of the artificial aortic valve.

Until now 25 cineradiographic studies in 20 patients with aortic valve prostheses have been performed.

For the cineradiography we have used a 5-in. image intensifier and a $35 \mathrm{~mm}$. camera. The recordings have been made in postero-anterior and right and left anterior oblique projections. In a few cases a true lateral projection has also been used.

\section{RESULTS}

In 17 of the 20 patients studied there have been no signs of abnormal function of the valve prosthesis. This has been confirmed by thoracic aortography in eight patients.

In these 17 patients the pattern of movement of the valve prosthesis was similar.

The movement of the prosthesis consisted of a small, spiral-like, 5 to 10 degrees twisting of the whole prosthesis around its axis. This was combined with a slight tilting of the prosthesis. Tilting was never more than 3 to 4 degrees in those 


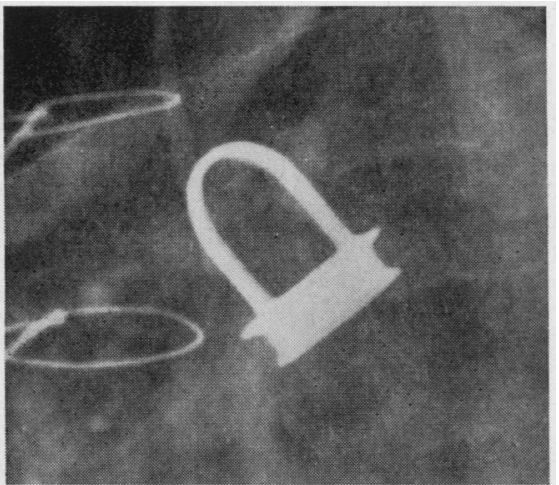

(a)

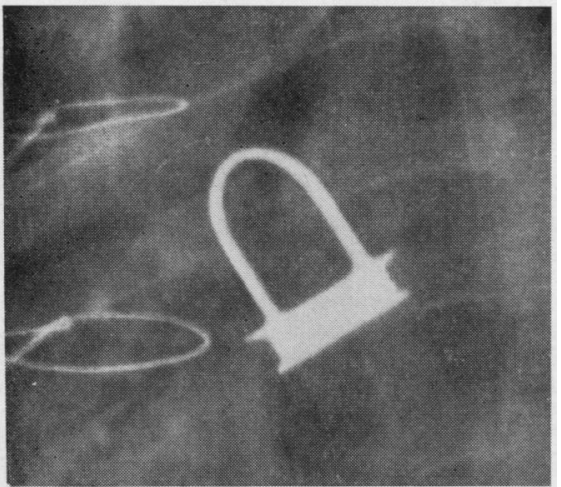

(b)

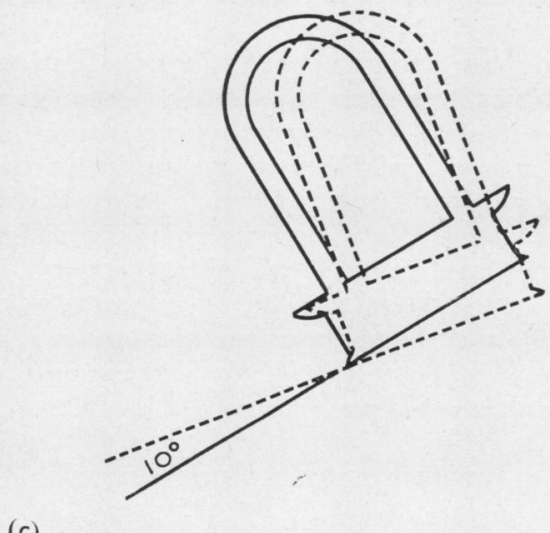

(c)

FIG. 2. Movements of a Starr ball valve prosthesis loose from its attachment on half the circumference. (a) Ventricular systole; (b) ventricular diastole; (c) diagram showing abnormal tilting of the prosthesis. patients who had no signs of abnormal function of the prosthesis.

In three patients an abnormal movement of the prosthesis was demonstrated by cineradiography (Fig. 2). In two of these patients there were clinical signs of aortic insufficiency, and this was confirmed by thoracic aortography (Fig. 1). In the third patient there was at first only an inconstant diastolic murmur. However, cineradiography showed abnormal movement of the prosthesis. A cineradiographic study repeated four weeks later showed some increase in this abnormal movement. At this time the clinical signs of aortic insufficiency were more definite. The patient died before a planned reoperation, and the prosthesis was found to be loose from its attachment over half the circumference.

In these three abnormal cases the most marked finding was an increase in the tilting of the prosthesis during the heart cycle. This tilting was typical when seen on the cinefilm. It usually amounted to about 10 degrees and occurred rapidly (Fig. 2). The spiral motion of the prosthesis was somewhat increased but to a much lesser degree.

In our limited experience, cineradiography seems to be a useful and simple method of controlling the Starr type aortic valve prosthesis postoperatively. The cineradiographic procedure causes the patient no discomfort and can be repeated as often as necessary.

An increase in the tilting of the valve prosthesis above 5 degrees seems to be pathognomonic of a loosening of the prosthesis from its attachment.

We now use cineradiography as a routine postoperatively in all patients to obtain a base-line study that can be used as a reference in follow-up examinations.

Fluoroscopy, using an image intensifier with or without TV systems, will provide the same information as cineradiography. The advantage of cineradiography is the objective record obtained of the movements of the valve prosthesis.

\section{SUMMARY}

In a series of 20 patients with an aortic Starr type valve prosthesis, cineradiography was used to assess the accuracy of the attachment of the prosthesis post-operatively. The method has helped to detect, and objectively to record, abnormal movements of the valve prosthesis. These abnormal tilting movements seem to be diagnostic of loosening of the aortic valve prosthesis from its attachment, with resulting aortic insufficiency. 